\title{
Vehicle Health Management Communications Requirements for AeroMACS
}

\author{
Robert J. Kerczewski \\ NASA Glenn Research Center \\ 21000 Brookpark Rd., MS 54-1 \\ Cleveland, OH 44135 \\ $+12164333434$ \\ Robert.J.Kerczewski@nasa.gov
}

\author{
Donna J. Clements \\ QinetiQ North America \\ 21000 Brookpark Rd., MS 500-AOS \\ Cleveland, OH 44135 \\ $+12164333566$ \\ donna.j.clements@nasa.gov
}

\author{
Rafael D. Apaza \\ NASA Glenn Research Center \\ 21000 Brookpark Rd., MS 54-1 \\ Cleveland, OH 44135 \\ $+12164337354$ \\ rafael.d.apaza@nasa.gov
}

\begin{abstract}
As the development of standards for the aeronautical mobile airport communications system (AeroMACS) progresses, the process of identifying and quantifying appropriate uses for the system is progressing. In addition to defining important elements of AeroMACS standards, indentifying the systems uses impacts AeroMACS bandwidth requirements. Although an initial $59 \mathrm{MHz}$ spectrum allocation for AeroMACS was established in 2007, the allocation may be inadequate; studies have indicated that $100 \mathrm{MHz}$ or more of spectrum may be required to support airport surface communications. Hence additional spectrum allocations have been proposed. Vehicle health management (VHM) systems, which can produce large volumes of vehicle health data, were not considered in the original bandwidth requirements analyses, and are therefore of interest in supporting proposals for additional AeroMACS spectrum. VHM systems are an emerging development in air vehicle safety, and preliminary estimates of the amount of data that will be produced and transmitted off an aircraft, both in flight and on the ground, have been prepared based on estimates of data produced by on-board vehicle health sensors and initial concepts of data processing approaches. This allowed an initial estimate of VHM data transmission requirements for the airport surface. More recently, vehicle-level systems designed to process and analyze VHM data and draw conclusions on the current state of vehicle health have been undergoing testing and evaluation. These systems make use of vehicle system data that is mostly different from VHM data considered previously for airport surface transmission, and produce processed system outputs that will be also need to be archived, thus generating additional data load for AeroMACS. This paper provides an analysis of airport surface data transmission requirements resulting from the vehicle level reasoning systems, within the context of overall VHM data requirements.
\end{abstract}

\section{TABle OF Contents}

1. INTRODUCTION ........................................... 1

2. AEROMACS OVERVIEW .................................. 2

3. VEHIClE HEAlth MANAgEMENT AND AEROMACS DATA REQUIREMENTS ................... 3

4. VEHICLE LEVEL REASONING SYSTEM............. 4

5. ANALYSIS OF VLRS COMMUNICATIONS REQUIREMENTS FOR AEROMACS ................... 5

6. CONCLUSION...................................................... 6

REFERENCES .................................................. 7

BIOGRAPHIES .................................................... 7

U.S. Government work not protected by U.S. copyright.

IEEEAC Paper \#1435, Version 3, Updated 17 January 2012.

\section{INTRODUCTION}

The Aeronautical Mobile Airport Communications System (AeroMACS) is a developing standard for wireless aeronautical communications for airport surface operations. AeroMACS support safety critical communications applications including air traffic control, airline operations, and advisory information [1]. A spectrum allocation for the AeroMACS network was established at the World Radiocommunications Conference (WRC) 2007. The allocation provides $59 \mathrm{MHz}$ of bandwidth in the 5091-5150 $\mathrm{MHz}$ band. Analyses of the bandwidth requirements for AeroMACS have shown that at least $100 \mathrm{MHz}$ of bandwidth will be needed in the long term. As a result, proposals for additional AeroMACS spectrum were submitted for consideration at the WRC-2012, in particular in the 5000$5030 \mathrm{MHZ}$ band. Whether or not all or some of this band is allocated for AeroMACS, the need for further refinement of AeroMACS long term bandwidth requirements must continue if further spectrum allocations are to be proposed for consideration at future WRCs.

The initial analyses of AeroMACS bandwidth requirements did not consider VHM data transmission, as the need had not yet been recognized. In future VHM systems now being researched by NASA and others, a large distribution of advanced on-board sensors will monitor many different aircraft systems while in flight. Vehicle level reasoning algorithms will analyze the sensor data and evaluate its meaning in order to determine if any action is required. Such a VHM system will accumulate and archive a large amount of data, much of which is of significance in terms of aircraft type and trend analysis regardless of whether any action was indicated by the VHM system during the flight and therefore must be offloaded from the aircraft. Initial estimates of VHM data offload requirements were developed to support the current WRC-12 AeroMACS spectrum proposals. As VHM system development has progressed, vehicle level reasoning systems (VLRS) have emerged as a component of the overall VHM concept, but present an additional data requirement as they will process vehicle data sets that are mostly different from the VHM sensor data previously analyzed.

This paper presents an analysis of the potential VLRS data 
transmission requirement for the airport surface. The following section provides an overview of AeroMACS and the current AeroMACS bandwidth requirements excluding VHM. That is followed by an overview of the vehicle health management concept and a review of recent analyses of VHM data requirements for AeroMACS. The next section describes a particular on-board vehicle health reasoning system currently under development and testing. The expected data archive requirement resulting from this system are then analyzed and the supporting data rates required from the AeroMACS airport surface communications system derived for typical airport sizes. The final section offers concluding remarks.

\section{AeromaCS Overview}

The Aeronautical Mobile Airport Communications System (AeroMACS) is a developing standard for wireless aeronautical communications restricted to the airport surface (stationary and mobile vehicles with wheels in contact with the airport surface). RTCA Special Committee 223 (SC223) and EUROCAE Working Group 82 (WG-82) are coordinating the development of the AeroMACS standards, after which international standardization will occur under the International Civil Aviation Organization (ICAO).

The AeroMACS network will operate within the 5091-5150 $\mathrm{MHz}$ band under the Aeronautical Mobile (Route) Service $(\mathrm{AM}(\mathrm{R}) \mathrm{S})$ allocation. This allocation was adopted at the 2007 World Radiocommunications Conference (WRC-07). The WRC-07 decision on Agenda Item 1.3 removed the prior limitation in the MLS extension band for "support of navigation/surveillance functions" only. The AM(R)S designation now allows communications services that support safety and regularity of flight applications. The protected allocation for aeronautical mobile services enables ICAO to approve international standards for wireless mobile communications networks on the airport surface. In the U.S. the AeroMACS network can be used for both mobile users and fixed assets that directly support safety and regularity of flight. AeroMACS services can be provided to aircraft anywhere on the airport surface, as long as wheels are in contact with the surface. AeroMACS can also be used for communications with a variety of service vehicles and airport infrastructure that directly support safety and regularity of flight[1].

RTCA SC-223 and EUROCAE WG-82 have developed the AeroMACS Profile draft published on December 15, 2010. The profile documents the adaptations required for the IEEE 802.16-2009 standard to provide wireless data communication service to fixed and mobile platforms in AeroMACS. Included in the profile is the channelization plan for AeroMACS, shown in Figure 1 [2]. The two standards groups are now working to develop the Minimum Operational Performance Standards, or MOPS.

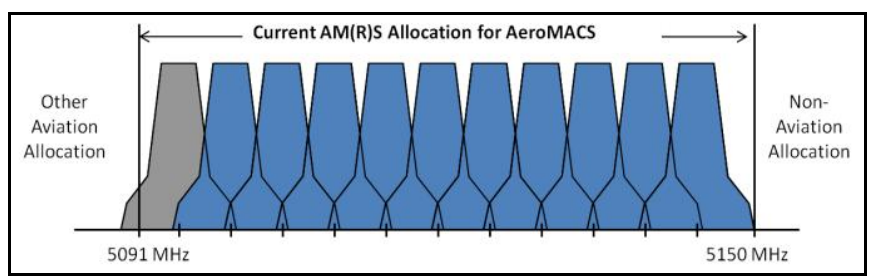

Figure 1 - AeroMACS Channel Plan: $5 \mathrm{MHz}$ channels over $5091-5150 \mathrm{MHz}$

User applications for transport over AeroMACS have been classified into the following five categories:

- Air Traffic Management (ATM) / Air Traffic Control (ATC)

- Aeronautical Information Management and Meteorological Data (AIM/MET)

- Owner/Operator

- Airport Authority

- Airport Infrastructure

The preliminary analysis of airport surface communications (previously referred to as the Airport Network and Location Equipment (ANLE) system) bandwidth requirements was prepared by the MITRE Corporation for the Federal Aviation Administration (FAA) in 2006, to support the proposal for an airport surface communications frequency allocation at WRC-2007[3]. This analysis was based in part on a airport surface communications requirements study communications commissioned by NASA Glenn Research Center in 2004[4]. The result of the analysis was a preliminary estimate of $60 \mathrm{MHz}$ of bandwidth required to support the airport surface requirement.

The MITRE analysis considered the following applications for the airport surface communications system:

- Sensor data

- Surveillance data

- Airport Surveillance Radar (ASR) data

- Airport Surface Detection Equipment Mode X (ASDE-X) data from remote units (RU) to the multi-processor

- ASDE-X display data

- Digital Bright Radar Indicator Tower Equipment (DBRITE) data

- Weather data

- Low Level Wind Shear Alert System (LLWAS) data

- Automated Weather Observing System (AWOS) / Automated Surface Observing

- System (ASOS) data

- Terminal Doppler Weather Radar (TDWR) data

- Integrated Terminal Weather System (ITWS) data

- Weather Systems Processor (WSP) data

- Navigation and landing aid data

- Terminal Navigational Aids (NAVAIDS) data 
- Automation data

○ Enhanced Traffic Management System (ETMS) data

- Center-TRACON Automation System (CTAS) data

- ATC voice (diversity path as a backup for existing facilities)

- Electronic Flight Bag (EFB) data

- Airport surface data transmission to taxiing aircraft and surface vehicles

In 2009, MITRE updated this analysis, including more detailed analysis of the OFDMA implementation of the IEEE 802.16e standard that is the basis of AeroMACS, to evaluate the bandwidth requirements at a large airport[5]. The analysis took into account long-term bandwidth requirements as envisioned under the Eurocontrol/FAA Communications Operating Concept and Requirements (COCR) for Future Radio System, which defines an initial future communications Phase 1 through the year 2020 and Phase 2 occurring beyond 2020[6]. For the Phase I time frame, the estimated bandwidth requirement is $100 \mathrm{MHz}$. For Phase II, the requirement is 110 to $120 \mathrm{MHz}$, depending on AeroMACS channel bandwidth.

Vehicle health management data requirements were not considered in these analyses.

\section{Vehicle Health Management AND AEROMACS DATA REQUIREMENTS}

NASA's research on vehicle health management originally took place under the Integrated Vehicle Health Management (IVHM) Project, and is now part of the Vehicle Systems Safety Technologies Project. These projects focus on reducing the impact of adverse events during flight. This long-term research includes the development of vehicle health sensors to detect and monitor the health of critical aircraft systems, as well as the algorithms that will interpret the resulting sensor data to determine the health status of the aircraft. Sensors for the monitoring of structural integrity, propulsion systems, pressure, gas, fire, and environmental effects are being developed to have a low weight, size, power and cost impact on the aircraft. They will be interconnected through an on-board wireless network to provide real time data for diagnosis, prognosis and mitigation algorithms intended to prevent and/or mitigate adverse events during aircraft flight. Adverse events include those that arise from system, subsystem, or component faults or failures due to damage, degradation, or environmental hazards that occur during flight.

The new IVHM capabilities will enable the rapid detection and diagnosis of these adverse events (in both the hardware and software) essential to the safe operation of the vehicle and will enable the estimation of the condition severity and the remaining useful life with confidence bounds for the affected system(s). Maintenance workers, crew, adaptive configuration systems and other control systems can take advantage of the estimated remaining useful life to enhance the safety profile of the aircraft. While in most cases the detected status will influence such things as vehicle maintenance cycles, in more time-critical cases, immediate action might be necessary to avoid serious safety issues.

The handling of the sensor data, between the point of generation at the sensor and the point of processing, will occur through an on-board wireless network combined with an off-board data communication link. Depending upon the criticality of the data and other factors, some sensorgenerated data will be processed on the aircraft, some will be stored for later download and analysis while the aircraft is on the ground and some will be delivered off-board while the aircraft is in flight.

In previous work, NASA estimated the total sensor data load for emerging vehicle health sensor networks and the subset of data required to be off-loaded from an aircraft during flight [7]. In this paper, NASA estimated the total sensors data output based on an Boeing 757-200 size aircraft and assessed requirements for off-board data communications while the aircraft was in flight. The analysis considered sensors and sensor categories related to NASA's IVMH Project research, including: Structural Integrity; Aircraft Systems and Avionics Health Monitoring; Aircraft Icing Sensing; Propulsion System Health Monitoring; Pressure Sensing; Weather Condition Sensing; Gas Sensing; Temperature Monitoring; and Smoke and Fire Detection. The requirements for off-board communications as developed in this approach are a maximum of $742.4 \mathrm{kbps}$. Of this total, $259.2 \mathrm{kbps}$ are real time off-board communications requirements, imposing maximum quality and availability requirements on the air-ground data communications link. A further $316.2 \mathrm{kbps}$ are near-real time requirements. The remaining $167 \mathrm{kbps}$ are delayed data requirements.

At the request of the FAA, this work was further extended in a white paper to provide an estimate of VHM data requirements on the airport surface [8]. In this initial analysis, the sensor data analyzed in [7] was subjected to further interpretation to estimate the amounts of sensor data that would be expected to be off-loaded from a future VHMequipped aircraft after landing. From [8]: The total data amount to be transmitted is roughly proportional to the flight time, or the time during which sensor data was being acquired. For example, assuming a 2 hour flight during which sensor data was being acquired for the entire duration, a total of $3.48 \mathrm{~Gb}$ of data would need to be transmitted off the aircraft on the airport surface. The transmission data rate required would then be dependent on the amount of time the aircraft is available on the surface. For example, an aircraft with a 30 minute turnaround time would required a data rate of $1.94 \mathrm{Mbps}$ to offload data from a 2 hour flight under this scenario.

In preparation for WRC-12, where proposals for additional AeroMACS spectrum in the 5000-5030 MHz band were submitted, additional analyses of VHM data offloading requirements on the airport surface were conducted, as reported to ICAO Aeronautical Communications Panel Working Group F[9]. It is important to explain the VHM data requirements currently being presented in support of the 
WRC-2012 AeroMACS spectrum proposal in order to provide context for the additional VLRS data requirement derived in Section 5. In the ICAO working paper analysis, data rates for COCR Phase 1 and 2 time frames were developed, and included two components: real time data requirements for aircraft while operating in the runway/taxiway/ramp areas (similar to the in-flight data offload requirements developed in [7]) and delayed data offload requirements for data that was acquired and stored during flight (as initially estimated in [8]). The analysis assumed a 2 hour flight, an 8 minute taxi and a 54 minute aircraft turn-around (COCR Phase 1) or a 36 minute aircraft turn-around (COCR Phase 2).

For the real time runway/taxiway/ramp component, the analysis assumed aircraft operating at maximum VHM data generation rate of $303.4 \mathrm{kbps} 10 \%$ of the time and at the minimum rate of $33.9 \mathrm{kbps} 90 \%$ of the time (rates derived from [7]). Assuming a maximum of 48 aircraft involved for COCR Phase 1 and 70 aircraft for Phase 2, the aggregate data rate that AeroMACS must support for this application is 2.98 Mbps for Phase 1 and 4.26 Mbps for Phase 2 (includes $8.05 \%$ data overhead).

For the offload of data stored during flight component, the analysis assumed aircraft operating at maximum VHM data storage of $483.2 \mathrm{kbps} 10 \%$ of the time and at the minimum rate of $5.7 \mathrm{kbps} 90 \%$ of the time (rates derived from [7]). Assuming a maximum of 154 aircraft downloading stored VHM data for COCR Phase 1 and 222 aircraft for Phase 2, the aggregate data rate that AeroMACS must support for this application is 17.22 Mbps for Phase 1 and $34.96 \mathrm{Mbps}$ for Phase 2.

The total AeroMACS data load due to VHM applications is 20.2 Mbps for Phase 1 and 39.22 Mbps for Phase 2.

To summarize, the previously derived and reported AeroMACS data loads resulting from VHM data offloading are:

- In-flight VHM communications [7]: $742.4 \mathrm{kbps}$ per aircraft.

- Airport surface VHM communications, initial estimate [8]: 1.94 Mbps.

- Airport surface VHM communications, extended analysis [9]: $20.2 \mathrm{Mbps}$ for Phase 1 and 39.22 Mbps for Phase 2.

Vehicle level reasoning is the next step in developing comprehensive VHM systems. Vehicle level reasoning system (VLRS) algorithms are under development to analyze various aircraft status indicators and health sensor data and evaluate its meaning in order to determine if any action is required while the vehicle is in flight. Eventually, complex systems are envisioned to evaluate the outputs of thousands of sensors on future aircraft.

However, initial versions of VLRS now under development and testing are relying on currently available sensors and aircraft system monitors. These VLRS have the potential to be implemented within the next several years, well before the advanced sensor systems being developed by NASA and others are available. The VLRSs will themselves be archiving a separate data set representing data gathered and analyzed on-board, as well as the resulting processed outputs. It is unknown to what extent future VLRS systems and advanced sensors systems will converge into a single system. Since previous estimates of aircraft VHM data offloading requirement on the airport surface were based primarily upon future VHM sensor installations, and there is a likelihood that the processing results will remain a separate entity from the sensor outputs and also require archiving, the VLRS data sets represent an additional AeroMACS data load requiring analysis.

VLRS is described in the next section, and the VLRS AeroMACS communications requirement follows.

\section{Vehicle LeVel Reasoning System}

The VLRS system is intended to prevent adverse events during flight by searching for potential system faults and analyzing the progression of faults and their severity. This is accomplished through a number of reasoning algorithms which make use of both current aircraft system monitoring data and historical data.

Two NASA Research Announcement awards were made for VLRS system research and development. Honeywell International, Inc. is developing the Vehicle Integrated Prognostic Reasoner (VIPR). VIPR consists of an on-board system analyzing the inputs from various aircraft sensors, as well as past history of the aircraft health [10]. The results of this work to date have provided a basis for Honeywell to estimate that the VIPR system will produce $2.76 \mathrm{MB}$ of VHM per flight hour that would be downloaded upon aircraft arrival.

The Boeing Company received the other NASA VLRS award. The Boeing system relies on the in-flight off-board communication of VHM data for processing on the ground. The progress to data has not yet been analyzed for revising or extending the in-flight off-board data requirements presented in [7].

From [10], VLRS addresses scenarios involving a fault or faults progressing in time and severity such that the effects are felt throughout the aircraft and its operations. For the VIPR development, the following categories are described:

1. Faults whose severity increases with time.

2. Binary repeating faults whose repetition increases with time.

3. Faults whose effects spread throughout the aircraft with time.

The VIPR system is based on Aircraft Diagnostic and Maintenance System and the Joint Strike Fighter Prognostic Health Management system as the prognostic engines. Interpretation of the prognostic outputs involves a multitude 
of time scales as well as coverage of aircraft systems and their interactions.

Data mining and machine learning techniques are use to characterize interactions between components and systems and the potential chain of events. The underlying algorithms establish the parametric relationships associated with the various entities in the VIPR fault propagation system model and discover new relationships from operational data.

As an on-board system intended to operate during flight, the flight crew is the primary consumer of VIPR outputs. A secondary set of consumers is the airline line maintenance and repair depot maintainers.

\section{ANALYSIS OF VLRS COMMUNiCATIONS REQUIREMENTS FOR AEROMACS}

The VIPR system described in the previous section is used as the model for estimating the VLRS communications requirements for AeroMACS. The VIPR concept is currently being evaluated using data from a fleet of 30 aircraft. The VIPR thus gathers inputs from currently available avionics diagnostics, sensors and system monitors.

As previously noted, the initial versions of VLRS will be gathering and processing data different from the types of advanced vehicle health sensor data considered for VHM data offloading requirements in [7-9]. As new vehicle health sensors are deployed, number of parameters monitored will increase. However, the data offload requirement resulting from these new sensors has been considered in the previous AeroMACS VHM requirements analyses.

VIPR operates by collecting $~ 182$ aircraft system health status parameters at varying sampling rates, using the ARINC 717 data recording format consisting of 512 12-bit word frame, one frame every second. This translates to 3600 frames, or $2.76 \mathrm{MB}$ per flight hour[11]. The figure of 2.76 MB per flight hour represents the best currently available figure used for deriving VLRS AeroMACS data loading.

Upon arrival of an aircraft on the airport surface, stored VLRS data will be downloaded for archiving. Using the figure of $2.76 \mathrm{MB}$ per flight hours, a maximum airport surface data rate estimate can be derived.

The estimate is dependent on the volume of flight operations at a given airport. In the ICAO working paper analysis discussed above, projected numbers based on COCR Phase 1 and 2 were used to derive estimates of maximum AeroMACS VHM data load.

Employing a similar method as in [9], namely assuming a two hour flight, an 8 minute taxi and a 54 minute aircraft turn-around (COCR Phase 1) or a 36 minute aircraft turnaround (COCR Phase 2), we can obtain, by dividing the total data load in bits by the taxi and turnaround time:

Phase 1:

$(2.76 \mathrm{MB} /$ hour $* 2$ hours $* 8 \mathrm{~b} / \mathrm{B}) \div(54+8 \mathrm{~min})=11.87 \mathrm{kbps}$
Phase 2:

$(2.76 \mathrm{MB} /$ hour $* 2$ hours $* 8 \mathrm{~b} / \mathrm{B}) \div(36+8 \mathrm{~min})=16.73 \mathrm{kbps}$

To obtain the aggregate AeroMACS VLRS loading due to all aircraft at an airport, the maximum aircraft numbers from COCR are used (154 for Phase 1, 222 for Phase 2):

Phase 1: $11.87 \mathrm{kbps}^{*} 154$ aircraft $=1.828 \mathrm{Mbps}$

Phase 2: 16.73 kbps $* 222$ aircraft $=3.713$ Mbps

These figures, while representing roughly an order of magnitude less of an AeroMACS data load than the VHM data estimated by the ICAO working paper [9], are nevertheless significant in terms of the total available AeroMACS bandwidth.

In order to compare the VLRS data offload requirement based on the COCR projections with current airport traffic density, we analyzed a typical medium sized airport, Cleveland Hopkins International Airport (CLE) and a very large airport, Chicago O'Hare International Airport (ORD). Using the published Monday flight schedule for CLE (June, 2010) and ETMS data for ORD (Monday, 14 June, 2010), the time length of arriving flights was tabulated for each 10 minute increment throughout the day (the total of the flight times for each flight arriving within a particular 10 minute increment). For example, three flights arriving in the ten minute time interval 00:40 through 00:50 that had flight times of 60 minutes, 90 minutes and 120 minutes respectively would yield a time length of arriving flights of 270 minutes. This provided a table of total flight arrival minutes. The total volume of VHM data to be downloaded during each 10 minute increment is calculated using the 2.76 MB per flight hour figure multiplied by $2.76 \mathrm{MB}$ per 60 minutes. Figures 2 and 3 show these results.

The peak airport surface data rate (in kbps) that must be supported for the VHM system based on the VIPR design is calculated by dividing the peak volume in MB by 600 seconds (per 10 minute interval), and multiplying by 8 bits per byte. For CLE, the peak data volume from Figure 2 is 66.47 MB. The resulting peak data rate required to support this data is $66.47 \mathrm{MB} * 8 \mathrm{~b} / \mathrm{B} \div 600 \mathrm{sec}=886.3 \mathrm{kbps}$. For ORD, the peak data volume from Figure 3 is $155.89 \mathrm{MB}$. The resulting peak data rate required at ORD is thus, 155.89 MB $* 8 / 600=2078.5 \mathrm{kbps}$. This peak data rate does not include network/protocol overhead or security overhead, and is based on June, 2010 traffic levels.

Thus, to support VLRS type VHM data downloading on the airport surface, AeroMACS will need to provide at least 1 Mbps of peak capacity for medium sized airports, and in excess of 2 Mbps for large airports for current airport traffic density. The data requirement derived for ORD in 2010 is very similar to the COCR Phase I calculation using the method in [9]. The COCR Phase 2 projection for beyond the year 2020 taking into account smaller aircraft turnaround times and a higher aircraft density increases the maximum data load by $79 \%$ compared to 2010 . 


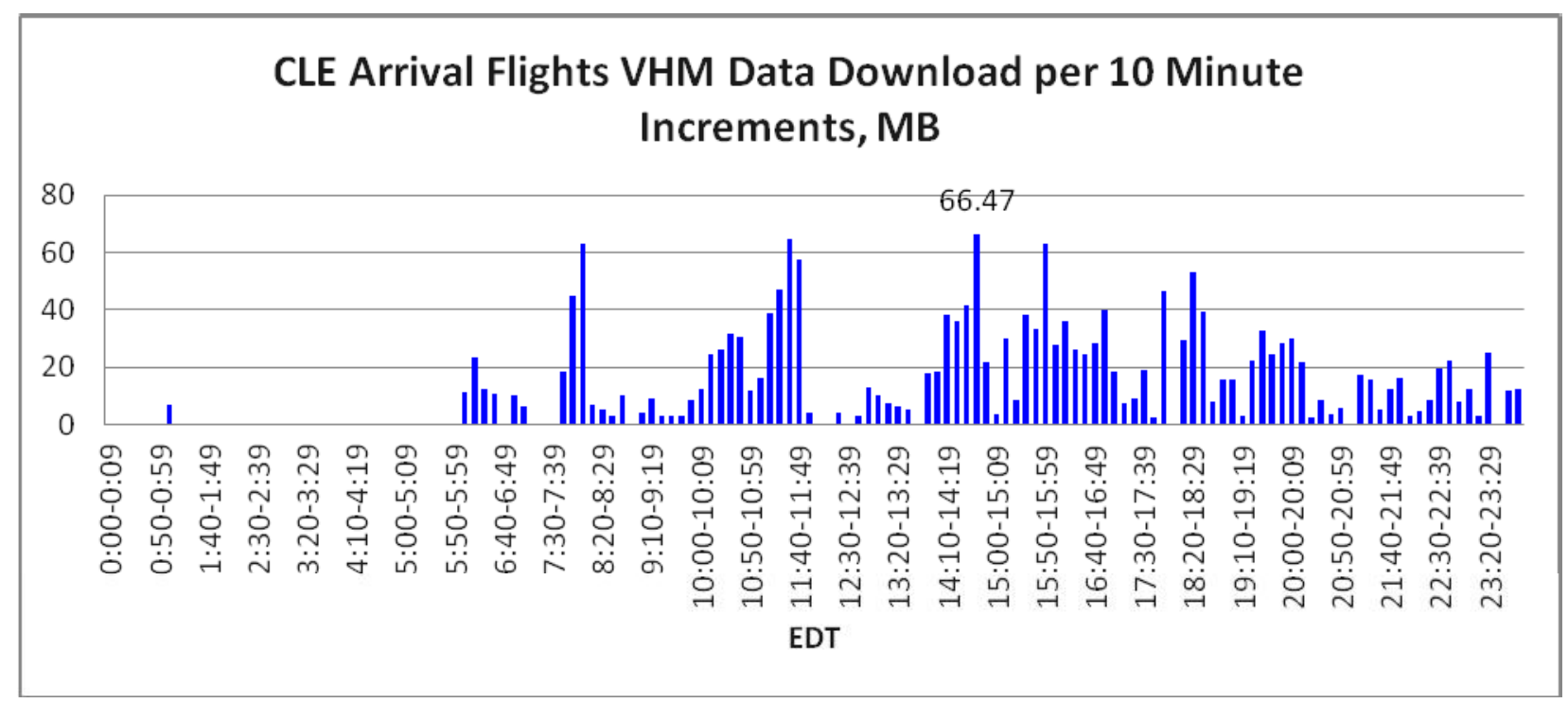

Figure 2 - Total VHM Data Download Requirements for CLE, MB per 10 minute Increment, June 2010

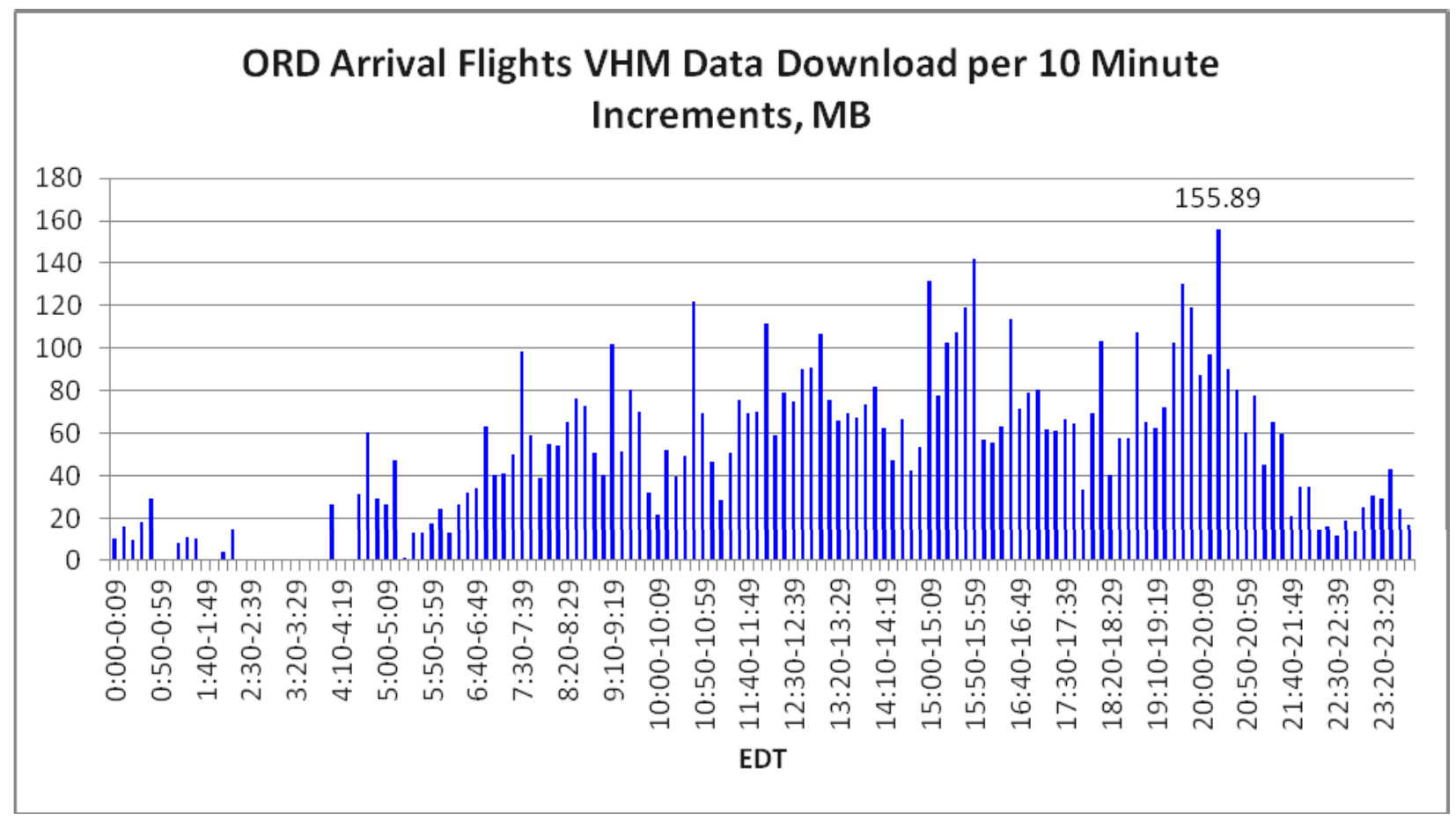

Figure 3 - Total VHM Data Download Requirements for ORD, MB per 10 minute Increment, June 2010

\section{Conclusion}

The AeroMACS airport surface communications system standards are currently under development. AeroMACS, based on the IEEE 801.16e standard, will provide wireless communications coverage for airport surface operations for safety related aviation applications. The WRC-2007 allocated $59 \mathrm{MHz}$ of bandwidth for AeroMACS. However, studies indicate that this allocation is inadequate to enable all of the projected AeroMACS applications. Hence, additional allocations are being proposed, requiring more detailed analysis of the data communications requirements of AeroMACS applications to support the allocation proposals.

Vehicle health management has been recognized as an important future AeroMACS application. Significant research and development work for VHM within the last decade will enable the future implementation of VHM systems. Aircraft VHM is being advanced through the development of advanced sensors as well as VLRSs to analyze the outputs of sensors and system monitors in real time to assess the current state of vehicle health and enable 
mitigating actions in case of detected faults and progressing failures which threaten the safety of the aircraft. The data produced by both on-board sensors and VLRS systems is of sufficient value for further analysis that it is anticipated to be archived in ground-based facilities for that purpose. This presents a significant data communications requirement for both in-flight and airport surface communications systems.

In this paper, we have presented the analyses of VHM airport surface data loads currently being used to support AeroMACS frequency allocation proposals. These analyses estimate a requirement of approximately $20 \mathrm{Mbps}$ in the near term (up to 2020) and approximately $40 \mathrm{Mbps}$ in the far term (beyond 2020). We then analyzed additional VHM AeroMACS data loading requirements resulting from the advent of VLRSs, and showed that including the VLRS data requirement increases the overall VHM AeroMACS data load requirement by approximately $10 \%$. This represents a significant additional requirement which must be considered in deriving the spectrum requirements for AeroMACS.

\section{REFERENCES}

[1] Budinger, James M. and Hall, "Aeronautical Mobile Airport Communications System (AeroMACS)", NASA Technical Memorandum 2011-217236, October 2011.

[2] Airport Surface Wireless Communications Profile Draft R1, RTCA SC-223, December 15, 2010.

[3] Gheorghisor, Izabela, Yan-Shek Hoh, "Preliminary Analysis of the Spectral Requirements of Future ANLE Networks", MP06W0000063, The MITRE Corporation, McLean, VA. March 2006.

[4] SAIC, September 2004, Task Order 2 Surface CNS Network Requirements - ACAST Final Report, SAIC for NASA.

[5] Gheorghisor, Izabela, "Spectral Requirements of Wireless Broadband Networks for the Airport Surface", 2009 ICNS Conference, Herndon, Virginia, May 2009.

[6] Eurocontrol/FAA, Communications Operating Concept and Requirements for Future Radio System, Version 2.0.

[7] Kerczewski, Robert J., and Kachmar, Brian A., "OffBoard Communications for Vehicle Health Management", 2010 IEEE Aerospace Conference, March 2010.

[8] Kerczewski, Robert J., "Off-Board Communications for Vehicle Health Management - Estimate of Off-Board Data Transmitted on Airport Surface", NASA Internal Memorandum, February 2010.

[9] Spectrum Requirements for Surface Applications at Airports in the $5 \mathrm{GHz}$ Range, ICAO Aeronautical Communications Panel Working Group F Meeting \#22, Working Paper 7, April 2010.

[10] Vehicle Integrated Prognostic Reasoner (VIPR) 2010 Annual Final Report, NASA Contractor Report NASA/CR-
2011-217147, May 2011.

[11] Correspondence with Dinkar Mylaraswamy, Honeywell Aerospace, September 2011.

\section{BIOGRAPHIES}

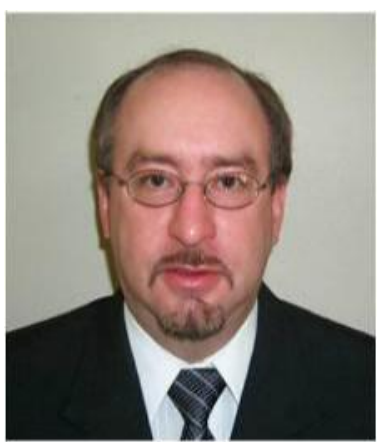

Robert J. Kerczewski has been involved with research and development communications systems for space and aeronautical applications for the Analex Corporation (1982-1986) and NASA (1986-present). $\mathrm{He}$ holds a BEE degree from Cleveland State University (1982) and an MSEE degree from Case Western Reserve University (1987). He is currently the Project Manager for the advanced CNS architectures and system technologies research.

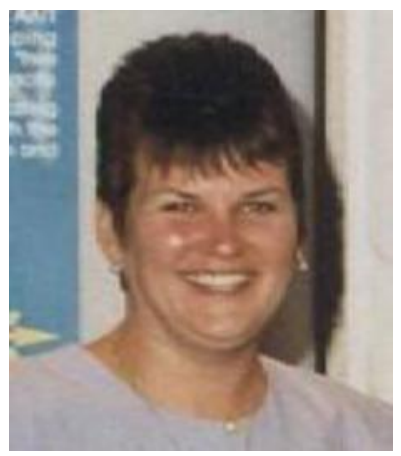

Donna J. Clements has worked as a contractor at the NASA Glenn Research Center for the past 27 years. She currently works for QinetiQ North America as a Project Specialist, supporting NASA's Federal Air Marshal Air Ground Communication System.

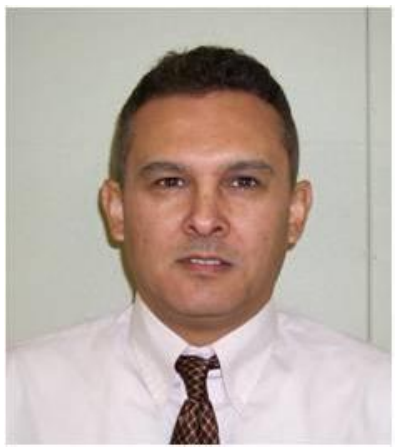

Rafael Apaza is a communications research engineer at NASA Glenn Research Center in the Architecture Branch. Prior to working for NASA, Rafael was the Communications Navigation and Surveillance (CNS) lead for the FAA Aviation Research and Development Office. Since 2002 he supported the Advanced CNS Architectures and System Technologies (ACAST) project for the NASA Glenn Research Center, leading the development of a surface wireless communications network for airports. In addition, Rafael supported the FAA's SWIM project, participating in both the SWIM Architecture Development and SWIM Transition projects. From 1999-2002 he was the FAA Great Lakes NAS Planning Program Manager for Michigan and Wisconsin. From 1987-1999, he worked as a systems engineer for FAA Airway Facilities, specializing in Communications and Surveillance. He holds a BSEE (1985), a MSEE (1995) from Wayne State University, and a MCIS (2001) from the University of Michigan. 